\title{
What are the priorities for prevention and control of non-communicable diseases and injuries in sub-Saharan Africa and South East Asia?
}

\begin{abstract}
Last year's UN high level meeting sought to galvanise the international community into scaling up its response to the escalating global burden of non-communicable diseases. With resources tight, D Chisholm and colleagues examine which interventions should be given priority for action and investment
\end{abstract}

\author{
D Chisholm health economist ${ }^{1}$, R Baltussen senior scientist ${ }^{2}$, D B Evans director ${ }^{1}$, G Ginsberg \\ health economist ${ }^{3}$, J A Lauer economist ${ }^{1}, \mathrm{~S}$ Lim associate professor ${ }^{4}$, M Ortegon researcher ${ }^{5}, \mathrm{~J}$ \\ Salomon associate professor $^{6}$, A Stanciole economist ${ }^{7}$, T Tan-Torres Edejer team coordinator ${ }^{1}$

\begin{abstract}
${ }^{1}$ Department of Health Systems Financing, World Health Organization, Geneva, Switzerland; ${ }^{2}$ Department of Primary and Community Care, Radboud University, Netherlands; ${ }^{3}$ Department of Medical Technology Assessment, Ministry of Health, Jerusalem, Israel; ${ }^{4}$ Institute for Health Metrics and Evaluation, University of Washington, Seattle, WA, USA; ${ }^{5}$ School of Medicine, Universidad del Rosario, Bogotá, Colombia; ${ }^{6}$ Department of Global Health and Population, Harvard School of Public Health, Boston, USA; ${ }^{7}$ World Bank, Washington, DC, USA
\end{abstract}

The millennium development goals adopted in 2000 laid down a core development agenda to which key international partners have since closely adhered, while a special session of the UN General Assembly held in 2001 on HIV/AIDS paved the way for a large and sustained international response to that emerging epidemic. ${ }^{1}$ Ten years on, high level delegates recently attended a second special session of the UN General Assembly on health, this time to discuss what to do about the rising epidemic of non-communicable diseases (NCDs) and how to integrate them into future development activities. ${ }^{2}$

A key issue for debate - both for delegates at the summit and for subsequent roll-out by countries - concerns the policies and strategies that could or should form the backbone of a renewed commitment to tackling non-communicable disease. Several criteria influence the selection of global or national strategies for prevention and control, including the current and projected burden of disease, cost effectiveness, fairness, and feasibility of implementing interventions, and political considerations. We have conducted a series of analyses focusing on cost effectiveness of interventions (see bmj.com). ${ }^{3-8}$ Here we draw together our findings with a view to identifying those strategies that offer the best value for money in tackling non-communicable diseases and injury in low income settings. The analyses complement an earlier $B M J$ series on the cost effectiveness of interventions focused on attainment of the millennium development goals. ${ }^{9}$

\section{Comparative analysis of value for money in health}

We have assessed the costs and effects of over 500 single or combined interventions for the prevention and control of non-communicable diseases and injuries in countries in sub-Saharan Africa and South East Asia that have high adult and child mortality. We have considered most of the leading contributors to this burden $^{10}$ : cardiovascular disease and diabetes, ${ }^{3}$ chronic respiratory diseases ${ }^{4}$ cancer, ${ }^{5}$ sensory loss disorders, ${ }^{6}$ mental disorders ${ }^{7}$ and road traffic injury. ${ }^{8}$

We used a generalised approach to estimating costs and modelling health effects; this has its strengths as well as limitations (box). On the cost side, we identified and measured resources used to provide an intervention then multiplied them by their respective price or unit cost; monetary values are expressed in international dollars (\$Int), which takes into account inter-regional differences in purchasing power (in the two resource settings considered here, one international dollar is around $\$ 0.32-\$ 0.44 ; £ 0.21-£ 0.28 ; € 0.25-€ 0.34)$. On the effect side, health gains at the population level are expressed in terms of disability adjusted life years (DALYs) averted, a measure 
that takes into account the effects on both mortality and morbidity.

When trawling through so many intervention strategies, how should we determine whether an intervention is cost effective? We defined an intervention as "highly cost effective" if the cost of generating an extra year of healthy life (equivalent to averting one DALY) was below the average annual income or gross domestic product (GDP) per person-that is, \$Int2000 in the two geographical sub-regions considered here. Interventions that produce a healthy life year for more than three times GDP per person (\$Int 6000) are considered "not cost-effective," and those in-between are considered cost effective. These thresholds are based on a recommendation of the WHO Commission on Macroeconomics and Health ${ }^{11}$ and broadly coincide with the thresholds used in several high income countries, including the United States and United Kingdom. ${ }^{12}$

\section{Priorities for intervention}

The table $\Downarrow$ provides an overview of the main individual and population based health improvement strategies that we assessed, grouped together into the analytical clusters or disease areas that we used in our analyses..$^{3-8}$ Interventions range from fiscal or regulatory measures that target the adverse health consequences of alcohol or tobacco use (such as increased taxes or advertising bans) to complex treatment strategies targeted at individuals with advanced stages of disease (including drug treatment for congestive heart failure and surgery for cancer).

The cost, effect, and cost effectiveness of assessed interventions in both regions varies by at least three orders of magnitude-for example, the annual cost of implementing an intervention varies from as little as a few cents to over \$Int10 per capita, while the annual health impact per million population ranges from 10 to 10000 healthy life years (see bmj.com). Likewise, average cost effectiveness ratios range from \$Int 14 to more than $\$ \mathrm{Int} 1 \mathrm{~m}$ (for a fruit and vegetable subsidy programme aimed at reducing the incidence of colorectal cancer). The median cost per health year gained is \$Int800-900. Results also vary between the two regions, most notably because of differences in risky health behaviours or rates of disease (for example, tobacco use and heart disease are more prevalent in South East Asia than in Africa).

By allowing all interventions to compete with each other for available resources, we can identify a subset that is dominant over the rest, starting with the single most cost effective option and then adding in the next most cost effective until the budget is used up (or until the value for money threshold is exceeded). The sequential incremental cost effectiveness ratios for these dominant interventions is listed in web appendix 2 (see bmj.com). Figures $1 \Downarrow$ and $2 \Downarrow$ show the most cost effective interventions grouped by disease or injury cluster for each region to illustrate how resources might be allocated within and across them.

\section{Do some health conditions fare better than others?}

Results for each of the disease clusters are discussed in greater detail in the accompanying research studies. ${ }^{3-8}$ If we look across them all we can see that almost all clusters have several interventions that are highly cost effective (that is, cost per DALY avoided is less than $\$$ Int2000); the exception is respiratory disorders, for which the most cost effective intervention is just over this threshold (low dose inhaled corticosteroids for mild asthma). So if decision makers in these resource settings accept this cost effectiveness threshold, these competing public health priority areas can all make some legitimate claim for scarce resources on economic efficiency grounds. If on the other hand decision makers were to use a threshold of only \$Int100 per DALY saved-a level proposed in the 1993 World Development Report, for example ${ }^{13}$

- virtually all of the interventions considered would not qualify for funding.

In terms of value for money, health conditions that are often left out of priority lists or action plans (such as sensory loss or mental disorders) have as much a case for funding as do cancer, cardiovascular disease, or diabetes. ${ }^{13}$ For example, antibiotic treatment of chronic otitis media (a persistent inflammation of the middle ear) is the most cost effective intervention in the two regions (< \$Int100/DALY saved), while extraction of cataracts and proactive screening for hearing loss are among the biggest potential contributors to population health gain.

Another striking observation is the degree of variation within health conditions. While some interventions offer very good value for money - drug treatment for hypertension, tobacco control measures, and epilepsy treatment, to name just a few-others within the same cluster do not. This is well illustrated by cancer interventions, where cost effectiveness ranges from a few hundred international dollars per DALY saved (for cervical cancer screening or lesion removal as required) to tens of thousands (such as some of the combined screening and treatment strategies for cervical and colorectal cancer). Other examples of interventions that are relatively cost ineffective include the use of newer antipsychotic drugs for schizophrenia and the treatment of advanced stages of chronic obstructive pulmonary disease. This points to the importance of carefully evaluating and choosing an appropriate set of interventions for scaled-up investment and implementation; selecting an inefficient set will waste money and limit potential health gains. ${ }^{14}$

\section{Is prevention better than treatment?}

It is often asserted that preventing disease will be a better bet than treating it after it has occurred. Our regional cost effectiveness analyses- together with the findings of Salomon et al in relation to care and prevention of non-communicable diseases in Mexico ${ }^{15}$ — show that population based preventive strategies do not clearly win out against more individual based interventions, implying that a multifaceted and balanced approach to the burden of non-communicable disease and injury is required. Furthermore, although the estimated cost of many preventive strategies is low ( $<\$$ Int 0.50 per capita)—particularly mass media and fiscal or regulatory measures that require no healthcare resources - others are very expensive. For example, annual screening of schoolchildren and adults for hearing loss is estimated to cost more than \$Int10 per person, and multidrug therapy for people with at least a 5\% risk of a cardiovascular disease event over the next 10 years would cost over \$Int2.50 per capita.

\section{Conclusion}

Through our analyses we have attempted to better understand the expected health effect and cost of a wide array of interventions that could be introduced or scaled up to combat the challenge of non-communicable diseases and injury in developing regions of the world. Although costs and effects vary substantially, there is a subset of interventions for all the health categories considered that offers good value for money and can form the basis of an evidence based and integrated 


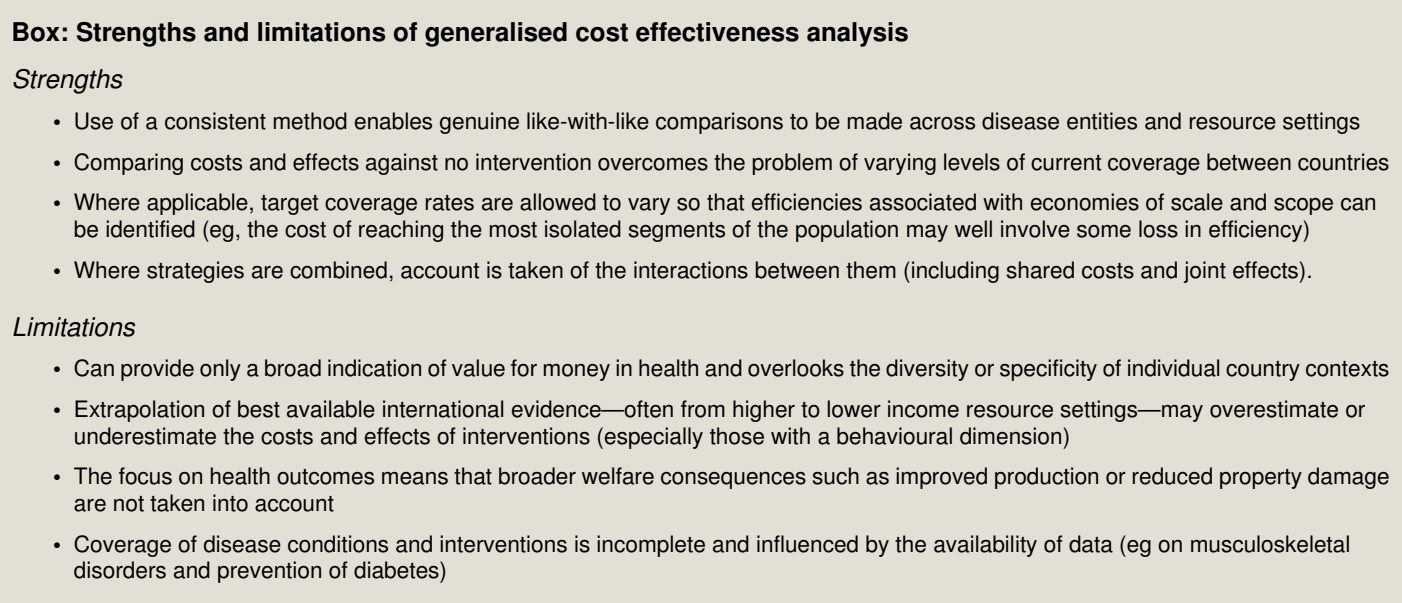

response. Some results are similar to those of previous analyses, ${ }^{16}{ }^{17}$ but we have included previously under-researched areas of economic evaluation (such as sensory loss disorder and road traffic injury) and are able to base our findings on validated and consistent methods. That said, our approach has some limitations, and gaps in our knowledge base remain (box). These gaps can be closed through the further development and application of economic modelling studies. Country level contextualisation studies-as carried out in Mexico, Estonia, Nigeria, and Vietnam, for example ${ }^{1518-20}$-are a worthwhile if resource intensive means of incorporating local priorities, health system characteristics, and data into the evaluative process. ${ }^{21}$

A final point is that efficiency represents but one of many criteria that need to be taken into account when allocating resources or determining priorities. In particular, efficient allocation of resources may not be fair. For example, treatment of schizophrenia may not be one of the most cost effective options, but withholding effective care for this severe, debilitating condition is likely to be considered highly unjust. This speaks to the need for careful and inclusive deliberation about how to develop health systems in a way that ensures not only good value for money but also equal access and financial protection for those in need (including poor and vulnerable people).

We thank Michele Sechi-Gatta for technical help and support in the preparation of summary tables and analyses.

Competing interests: All authors have completed the ICMJE uniform disclosure form at www.icmje.org/coi_disclosure.pdf (available on request from the corresponding author) and declare: no support from any organisation for the submitted work; no financial relationships with any organisations that might have an interest in the submitted work in the previous three years, no other relationships or activities that could appear to have influenced the submitted work.

Contributors and sources: All authors are or were staff members of WHO and each has been closely involved in the development and application of sectoral cost effectiveness analysis, specifically via WHO's Choosing Interventions that are Cost-Effective (CHOICE) work programme. The findings, interpretations, and conclusions in this paper do not necessarily represent the views of WHO. DC drafted and revised the paper; all other authors contributed to the design and data composition as well as commentaries on successive drafts. DC and TTTE act as guarantors.
Provenance and peer review: Not commissioned; externally peer reviewed.

1 United Nations. Declaration of commitment on HIV/AIDS. 2001. http://data.unaids.org/ publications/irc-pub03/aidsdeclaration_en.pdf.

2 United Nations. Political declaration of the high-level meeting of the general assembly on the prevention and control of non-communicable diseases (document A/66/L.1). UN, 2011.

3 Ortegon M, Lim S, Chisholm D, Mendis S. Cost effectiveness of strategies to combat cardiovascular disease, diabetes and tobacco use in sub-Saharan Africa and South East Asia: mathematical modelling study. BMJ 2012;344:e607.

4 Stanciole A, Ortegon M, Chisholm D, Lauer J. Cost effectiveness of strategies to combat chronic respiratory diseases in sub-Saharan Africa and South East Asia: mathematical modelling study. BMJ 2012;344:e608.

5 Ginsberg G, Lauer J, Zelle S, Baeten S, Baltussen R. Cost effectiveness of strategies to combat breast, cervical and colorectal cancer in sub-Saharan Africa and South East Asia: mathematical modelling study. BMJ 2012;344:e614.

6 Chisholm D, Saxena S. Cost effectiveness of strategies to combat neuropsychiatric conditions in sub-Saharan Africa and South East Asia: mathematical modelling study. BMJ 2012;344:e609.

7 Baltussen R, Smith A. Cost effectiveness of interventions to combat vision and hearing loss in sub-Saharan Africa and South East Asia: mathematical modelling study. BMJ 2012;344:e615.

8 Chisholm D, Naci H, Hyder AA, Tran NT, Peden M. Cost effectiveness of strategies for reducing road traffic injuries in sub-Saharan Africa and South East Asia: mathematical modelling study. BMJ 2012;344:e612.

9 Evans DB, Lim S, Adam T, Tan-Torres Edejer T, WHO CHOICE MDG Team. Evaluation of current strategies and future priorities for improving health in developing countries. BMJ 2005;331:1457-61.

10 WHO. The global burden of disease; 2004 update. WHO, 2008

11 WHO Commission on Macroeconomics and Health. Macroeconomics and health: investing in health for economic development. WHO, 2001.

12 Eichler HG, Kong S, Gerth WC, Mavros P, Jönsson B. Use of cost effectiveness analysis in health-care resource allocation decision-making: how are cost-effectiveness thresholds expected to emerge? Value in Health 2004;7:518-28.

13 WHO. 2008-2013 action plan for the global strategy for the prevention and control of noncommunicable diseases. WHO, 2008.

14 WHO. World health report 2010. Health system financing; the path to universal coverage . 2010. www.who.int/whr/2010/en/index.html.

15 Salomon JA, Carvalho N, Gutierrez-Delgado C, Casey A, Hogan DR, Lee D, et al. Intervention strategies to reduce burden of non-communicable diseases in Mexico: cost effectiveness analysis. BMJ 2012;344:e355.

16 Gaziano TA, Galea G, Reddy KS. Scaling up interventions for chronic disease prevention the evidence. Lancet 2007;370:1939-46.

17 Jamison DT, Breman JG, Measham AR, Alleyne G, Claeson M, Evans DB, et al, eds. Disease control priorities in developing countries. 2nd ed. Oxford University Press, World Bank, 2006.

18 Lai T, Habicht J, Reinap M, Chisholm D, Baltussen R. Costs, health effects and cost-effectiveness of alcohol and tobacco control strategies in Estonia. Health Policy 2007;84:75-88.

19 Gureje O, Chisholm D, Kola L, Lasebikan V, Saxena S. Cost-effectiveness of an essential mental health intervention package in Nigeria. World Psychiatry 2007;6:42-8.

$20 \mathrm{Ha}$ DA, Chisholm D. Cost-effectiveness analysis of interventions to prevent cardiovascular disease in Vietnam. Health Policy Plan 2011;26:210-22.

21 Hutubessy R, Chisholm D, Edejer TTT, WHO-CHOICE. Generalized cost-effectiveness analysis for national-level priority-setting in the health sector. Cost-effectiveness and Resource Allocation 2003;1:8.

Cite this as: BMJ 2012;344:e586

(c) BMJ Publishing Group Ltd 2012 


\section{Table}

\begin{tabular}{|c|c|c|c|}
\hline \multirow[b]{2}{*}{ Cluster } & \multirow[b]{2}{*}{ Health conditions } & \multicolumn{2}{|c|}{ Main intervention strategies } \\
\hline & & Population & Individual \\
\hline \multirow[t]{3}{*}{ Cancer } & Breast cancer & Biannual mammographic screening & Mastectomy, radiotherapy, chemotherapy \\
\hline & Cervical cancer & Screening (VIA, smear test); HPV vaccination & Lesion removal, radiotherapy, chemotherapy, surgery \\
\hline & Colorectal cancer & $\begin{array}{l}\text { Screening (faecal occult blood testing, } \\
\text { colonoscopy) }\end{array}$ & $\begin{array}{l}\text { Polyps/lesion removal, radiotherapy, chemotherapy, } \\
\text { surgery }\end{array}$ \\
\hline \multirow[t]{4}{*}{$\begin{array}{l}\text { Cardiovascular } \\
\text { disease }\end{array}$} & Hypertension and cholesterolaemia & $\begin{array}{l}\text { Reduced dietary salt (regulated food industry), } \\
\text { mass media campaigns to reduce cholesterol }\end{array}$ & $\begin{array}{l}\text { Blood pressure and cholesterol lowering drugs, } \\
\text { combined drug therapy for those at high risk }\end{array}$ \\
\hline & Ischaemic heart disease and stroke & & $\begin{array}{l}\text { Single or combined drug regimens for } \\
\text { acute and post-acute heart disease and stroke }\end{array}$ \\
\hline & Diabetes mellitus & & $\begin{array}{l}\text { Glycaemic control, retinopathy/neuropathy screening } \\
\text { and treatment }\end{array}$ \\
\hline & Tobacco use & Excise taxes, advertising bans; indoor air laws & Nicotine replacement, counselling \\
\hline $\begin{array}{l}\text { Respiratory } \\
\text { disorders }\end{array}$ & $\begin{array}{l}\text { Asthma; chronic obstructive } \\
\text { pulmonary disease }\end{array}$ & & $\begin{array}{l}\text { Inhaled bronchodilators and corticosteroids, smoking } \\
\text { cessation, treatment of exacerbations }\end{array}$ \\
\hline $\begin{array}{l}\text { Sensory loss } \\
\text { disorders }\end{array}$ & $\begin{array}{l}\text { Trachoma; cataract; refractive error; } \\
\text { hearing loss; chronic otitis media }\end{array}$ & Active and passive screening strategies & $\begin{array}{l}\text { Antibiotic treatment. trichiasis surgery, cataract } \\
\text { extraction }\end{array}$ \\
\hline \multirow[t]{3}{*}{ Mental disorders } & Psychotic and affective disorders & & Psychoactive drugs, psychosocial therapies \\
\hline & Epilepsy & & Antiepileptic drugs \\
\hline & Hazardous alcohol use & $\begin{array}{l}\text { Excise taxes, advertising bans, restricted } \\
\text { access }\end{array}$ & Brief physician advice \\
\hline Injury & Road traffic injury & $\begin{array}{l}\text { Enforcement of drink-driving laws, speed } \\
\text { limits, seat belt use, helmet use }\end{array}$ & \\
\hline
\end{tabular}

$\mathrm{VIA}=$ visual inspection with acetic acid; HPV=human papillomovirus. 


\section{Figures}

\section{Sensory disorders}

Chronic otitis media (COM-2: Topical antibiotics, 50\% coverage)

Trachoma (TRC-11: Trichiasis surgery, $80 \%$ coverage)

Cataract (CTR-4: Extracapsular cataract extraction with posterior chamber lens implant, 80\% coverage) Refractive error (RE-9: Annual screening of all primary and secondary schoolchildren, $80 \%$ coverage) Meningitis (MEN-1: Ceftriaxione, $50 \%$ coverage)

Hearing loss (HEA-6: Passive screening of all children and adults, $50 \%$ coverage)

Hearing loss (HEA-4: Screening of adults every 5 years, $50 \%$ coverage)

Hearing loss (HEA-23: Annual screening of all schoolchildren + adults every 5 years, $80 \%$ coverage)

Trachoma (TRC-20: Mass treatment azithromycin + thrichiasis surgery, $95 \%$ coverage)

\section{Cancers}

Cervical cancer (CVC-129: PAP smear at age 40 with lesion removal + cancer treatment, $50 \%$ coverage) Colorectal cancer (CRC-35: Treatment: surgery and/or chemotherapy and/or radiotherapy, $80 \%$ coverage) Cervical cancer (CVC-4: Treatment: surgery and/or chemotherapy and/or radiotherapy, $95 \%$ coverage) Cervical cancer (CVC-51: PAP smear at age $40+$ waning HPV at age 12 ( $\$ 0.60$ per dose $)+$ treatment, $95 \%$ coverage) Cervical cancer (CVC-35: VIA at age $40+$ waning HPV at age $12(\$ 0.60$ per dose $)+$ treatment, $95 \%$ coverage) Cervical cancer (CVC-49: VIA $(35,40,45)+$ waning HPV at age 12 (\$0.60 per dose) + treatment, $95 \%$ coverage) Breast cancer (BRC-6: Optimal programme, $50 \%$ coverage)

Colorectal cancer (CRC-18: Colonoscopy at age 50 and surgical removal of polyps + treatment, $95 \%$ coverage) Cervical cancer (CVC-37: PAP $(5,20,65)+$ waning HPV at age 12 ( $\$ 0.60$ per dose $)+$ treatment, $95 \%$ coverage) Colorectal cancer (CRC-14: Colonoscopy every 10 years and surgical re moval of polyps + treatment, $95 \%$ coverage) Cervical cancer (CVC-34: PAP $(5,20,65)+$ waning HPV at age $12(\$ 0.60$ per dose $)+$ treatment, $95 \%$ coverage $)$ Colorectal cancer (CRC-15: Sigmoidoscopy ( 5 yearly) + annual FOB + removal of polyps + treatment, $95 \%$ coverage) Cervical cancer (CVC-33: PAP $(1,20,65)+$ waning HPV at age 12 ( $\$ 0.60$ per dose) + treatment, $95 \%$ coverage) Cervical cancer (CVC-39: PAP $(1,20,30)$ and PAP/HPV $(1,30,65)+$ waning HPV + treatment, $95 \%$ coverage)

\section{Cardiovascular disease (CVD), diabetes, and tobacco use}

CVD (11: Preventive multidrug treatment $335 \%$ risk of CVD event)

CVD (77: Preventive multidrug treatment $35 \%$ risk + multidrug treatment of post acute IHD and stroke + diuretics/exercise for CHF) CVD (78: Preventive multidrug treatment $>35 \%$ risk + multidrug treatment of acute $\mathrm{Ml}+$ post acute IHD and stroke + diuretics/exercise for $\mathrm{CHF}$ ) Tobacco (TOB-2: Increased taxation)

Diabetes (DM-4: Retinopathy screening + photocoagulation, $80 \%$ coverage)

CVD (73: Preventive multidrug treatment $>25 \%$ risk + multidrug treatment of acute $\mathrm{MI}+$ post acute IHD and stroke + diuretics/exercise for CHF) Tobacco (TOB-15: Tax increase + ad ban + clean indoor air)

Tobacco (TOB-27: Tax increase + ad ban + clean indoor air + information/labelling)

CVD (8: Preventive multidrug treatment $>5 \%$ risk of CVD event)

Diabetes (DM-6: Standard glucose control + retinopathy, $80 \%$ coverage)

Diabetes (DM-8: Intensive glucose control + retinopathy, $80 \%$ coverage)

Tobacco (TOB-36: Tax increase + ad ban + clean indoor air + information/labelling + counselling)

\section{Respiratory disorders}

Asthma (AST-1: Low dose inhaled cortocosteroids for mild cases, $80 \%$ coverage)

Asthma (AST-2: Low dose inhaled cortocosteroids + long acting $\beta$ agonists for moderate cases, $80 \%$ coverage)

Chronic obstructive pulmonary disease (COPD-3: Inhaled bronchodilator (stage II) $80 \%$ coverage)

\section{Mental disorders}

Alcohol (ALC-3: Increased taxation (current $+50 \%$ ))

Alcohol (ALC-9: Increased tax and scaled up tax enforcement)

Alcohol (ALC-15: Increased tax + reduced access + tax enforcement)

Epilepsy (EPI-1: Older anti-epileptic drug in primary care at $50 \%$ coverage)

Epilepsy (EPI-2: Older anti-epileptic drug in primary care at $80 \%$ coverage)

Depression (DEP-2: Episodic treatment: newer antidepressant drug (SSRIs), 50\% coverage)

Depression (DEP-7: Maintenance psychosocial treatment + newer antidepressant drug, 50\% coverage)

Bipolar disorder (BIP-1: Older mood stabiliser drug (lithium), 50\% coverage)

Schizophrenia (SCZ-3: Older antipsychotic drug + psychosocial treatment, $80 \%$ coverage)

Bipolar disorder (BIP-2: Older mood stabiliser drug (lithium) + psychosocial care, $50 \%$ coverage)

Schizophrenia (SCZ-4: Newer antipsychotic drug + psychosocial treatment, $80 \%$ coverage)

\section{Injuries (road traffic)}

RTI-5: Legislation and enforcement of bicycle helmet use, $80 \%$ coverage

RTI-9: Speed cameras + breath testing + motorcycle helmets, $80 \%$ coverage

RTI-13: Seatbelts + motorcycle helmets + bicycle helmets + speed cameras + breath testing, $80 \%$ coverage

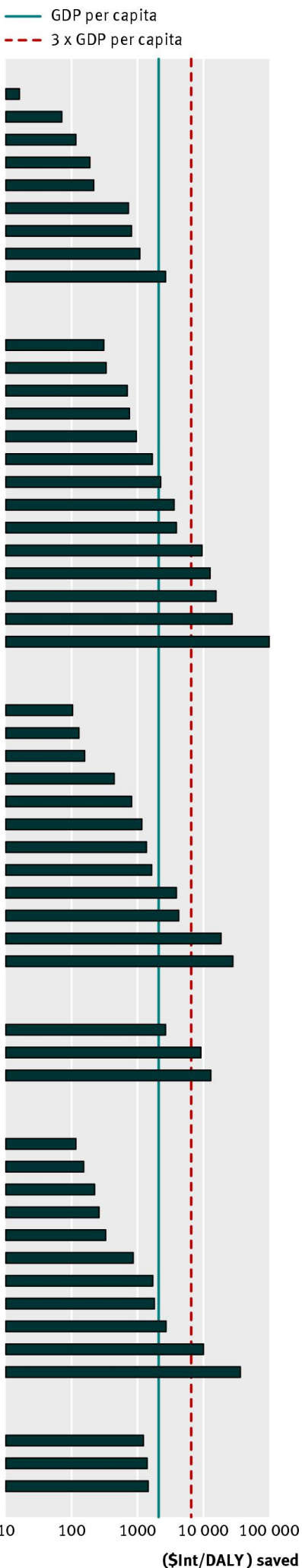

Fig 1 Incremental cost effectiveness (\$Int/DALY saved) of dominant interventions in sub-Saharan African countries with high child and adult mortality (HPV=human papillomavirus, $\mathrm{VIA}=$ visual inspection with acetic acid, IHD=ischaemic heart disease, $\mathrm{CHF}=$ chronic heart failure, $\mathrm{FOB}=$ faecal occult blood) 


\section{Sensory disorders}

Chronic otitis media (COM-2: Topical antibiotics, 50\% coverage)

Meningitis (MEN-1: Ceftriaxione, $50 \%$ coverage)

Cataract (CTR-6: Extracapsular cataract extraction with posterior chamber lens implant, 95\% coverage)

Refractive error (RE-2: Annual screening of all secondary schoolchildren, $50 \%$ coverage)

Refractive error (RE-9: Annual screening of all primary and secondary schoolchildren, $80 \%$ coverage)

Trachoma (TRC-11: Trichiasis surgery, $80 \%$ coverage)

Hearing loss (HEA-6: Passive screening of all children and adults, $50 \%$ coverage)

Hearing loss (HEA-11: Annual screening of all schoolchildren + adults every 5 years, $50 \%$ coverage)

Trachoma (TRC-20: Mass treatment azithromycin + thrichiasis surgery, 95\% coverage)

\section{Cancers}

Cervical cancer (CVC-129: PAP smear at age 40 with lesion removal + cancer treatment, $50 \%$ coverage)

Colorectal cancer (CRC-10: Treatment: surgery and/or chemotherapy and/or radiotherapy, $95 \%$ coverage)

Cervical cancer (CVC-4: Treatment: surgery and/or chemotherapy and/or radiotherapy, $95 \%$ coverage)

Cervical cancer (CVC-23: PAP at age 40 with lesion removal + cancer treatment, 95\% coverage)

Cervical cancer (CVC-7: VIA at age 40 with lesion removal + cancer treatment, $95 \%$ coverage)

Cervical cancer (CVC-21: VIA $(35,40,45)$ with lesion removal + cancer treatment, $95 \%$ coverage)

Breast cancer (BRC-12: Optimal programme, $50 \%$ coverage)

Colorectal cancer (CRC-17: Sigmoidoscopy at age 50 and surgical removal of polyps + treatment, $95 \%$ coverage)

Cervical cancer (CVC-34: PAP $(5,20,65)+$ waning HPV at age 12 ( $\$ 0.60$ per dose) + treatment, $95 \%$ coverage)

Colorectal cancer (CRC-14: Colonoscopy every 10 years and surgical removal of polyps + treatment, $95 \%$ coverage)

Cervical cancer (CVC-41: PAP $(3,20,30)$ and PAP/HPV $(3,30,65)+$ waning HPV $(\$ 0.60$ per dose $)+$ treatment, $95 \%$ coverage $)$

Colorectal cancer (CRC-15: Sigmoidoscopy ( 5 yearly) + annual FOB + removal of polyps + treatment, $95 \%$ coverage)

Cervical cancer (CVC-39: PAP $(1,20,30)$ and PAP/HPV $(1,30,65)+$ waning HPV + treatment, $95 \%$ coverage)

\section{Cardiovascular disease (CVD), diabetes, and tobacco use}

CVD (42: Treatment of CHF with diuretics)

Tobacco (TOB-2: Increased taxation)

CVD (11: Preventive multidrug treatment $335 \%$ risk of CVD event)

CVD (77: Preventive multidrug treatment $35 \%$ risk + multidrug treatment of post acute IHD and stroke + diuretics/exercise for CHF)

Tobacco (TOB-10: Tax increase + clean indoor air)

Diabetes (DM-4: Retinopathy screening + photocoagulation, $80 \%$ coverage)

Tobacco (TOB-15: Tax increase + ad ban + clean indoor air)

Tobacco (TOB-27: Tax increase + ad ban + clean indoor air + information/labelling)

CVD (78: Preventive multidrug treatment $335 \%$ risk + multidrug treatment of acute $\mathrm{MI}+$ post acute IHD and stroke + diuretics/exercise for CHF)

CVD (73: Preventive multidrug treatment $25 \%$ risk + multidrug treatment of acute $\mathrm{MI}+$ post acute IHD and stroke + diuretics/exercise for CHF) CVD (8: Preventive multidrug treatment $>5 \%$ risk of CVD event)

Diabetes (DM-6: Standard glucose control + retinopathy, $80 \%$ coverage)

Tobacco (TOB-35: Tax increase + ad ban + clean indoor air + information/labelling + brief advice)

Diabetes (DM-8: Intensive glucose control + retinopathy, $80 \%$ coverage)

\section{Respiratory disorders}

Asthma (AST-1: Low dose inhaled cortocosteroids for mild persistent asthma cases, $80 \%$ coverage)

Chronic obstructive pulmonary disease (COPD- 2 : Influenza vaccine, $80 \%$ coverage)

Asthma (AST-2: Low dose inhaled cortocosteroids + long acting $\beta$ agonists for moderate cases, $80 \%$ coverage)

Chronic obstructive pulmonary disease (COPD-3: Inhaled bronchodilator (stage II) $80 \%$ coverage)

\section{Mental disorders}

Epilepsy (EPI-2: Older antiepileptic drug in primary care at $80 \%$ coverage)

Depression (DEP-2: Episodic treatment: newer antidepressant drug (SSRIs), 50\% coverage)

Alcohol (ALC-3: Increased taxation (current + 50\%))

Depression (DEP-7: Maintenance psychosocial treatment + newer antidepressant drug, 50\% coverage)

Alcohol (ALC-10: Increased tax + reduced access)

Bipolar disorder (BIP-1: Older mood stabiliser drug (lithium), 50\% coverage)

Schizophrenia (SCZ-3: Older antipsychotic drug + psychosocial treatment, $80 \%$ coverage)

Alcohol (ALC-14: Increased tax + ad ban + reduced access)

Alcohol (ALC-15: Increased tax + reduced access + tax enforcement)

Alcohol (ALC-17: Increased tax + brief advice + ad ban + reduced access + tax enforcement)

Bipolar disorder (BIP-2: Older mood stabiliser drug (lithium) + psychosocial care, $50 \%$ coverage)

Schizophrenia (SCZ-4: Newer antipsychotic drug + psychosocial treatment, $80 \%$ coverage)

\section{Injuries (road traffic)}

RTI-12: Seatbelts + motorcycle helmets + speed cameras + breath testing, $80 \%$ coverage

RTI-13: Seatbelts + motorcycle helmets + bicycle helmets + speed cameras + breath testing, $80 \%$ coverage

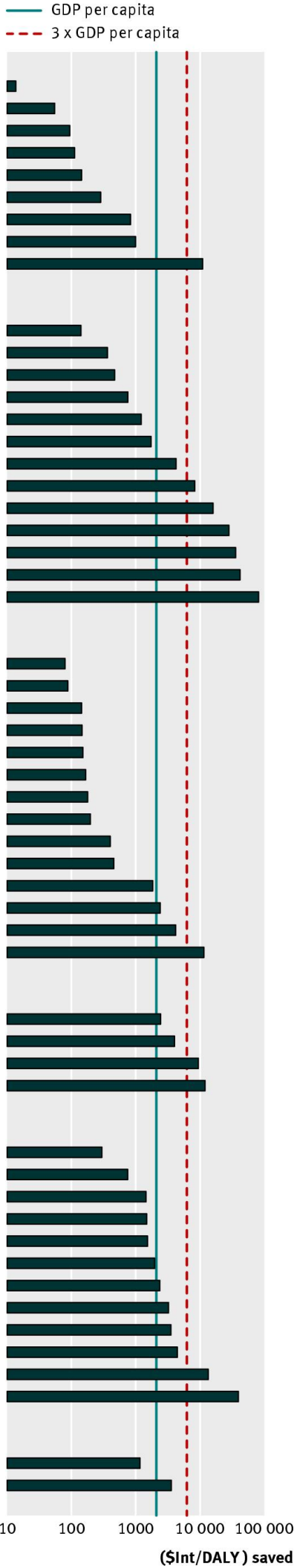

Fig 2 Incremental cost effectiveness (\$Int/DALY saved) of dominant interventions in South East Asian countries with high child and adult mortality (HPV=human papillomavirus, VIA= visual inspection with acetic acid, IHD=ischaemic heart disease, $\mathrm{CHF}=$ chronic heart failure, $\mathrm{FOB}=$ faecal occult blood) 